\title{
Acute Intermittent Porphyria
}

National Cancer Institute

\section{Source}

National Cancer Institute. Acute Intermittent Porphyria. NCI Thesaurus. Code C84536.

A genetic metabolic disorder inherited in an autosomal dominant pattern. It is caused by a deficiency of the enzyme porphobilinogen deaminase, which is involved in heme biosynthesis. Signs and symptoms include nausea, vomiting, severe abdominal pain and distension, urinary retention, port-wine urine discoloration, hypertension, tachycardia, muscle weakness, loss of sensation, anxiety, depression, and arm, leg and back pain. 